\title{
Developmental continuity and change in responses to social and nonsocial categories in human extrastriate visual cortex
}

\author{
Kevin A. Pelphrey ${ }^{1 *}$, Juliana Lopez ${ }^{2}$ and James P. Morris ${ }^{3}$ \\ Yale Child Study Center, Yale University School of Medicine, Yale University, New Haven, CT, USA \\ 2 University of Illinois, Chicago School of Medicine, University of Illinois at Chicago, Chicago, IL, USA \\ ${ }^{3}$ Department of Psychology, University of Virginia, Charlottesville, VA, USA
}

\author{
Edited by: \\ Silvia A. Bunge, University of California, \\ USA

\section{Reviewed by:} \\ Charles A. Nelson, Harvard University, \\ USA \\ Andy Calder, Cambridge University, UK \\ *Correspondence: \\ Kevin A. Pelphrey, Yale Child Study \\ Center, Yale University, 230 South \\ Frontage Road, New Haven, CT 06520, \\ USA. \\ e-mail: kevin.pelphrey@yale.edu
}

It is well known that adult human extrastriate visual cortex contains areas that respond in a selective fashion to specific categories of visual stimuli. Three regions have been identified with particular regularity: the fusiform face area (FFA), which responds to faces more than to other objects; the parahippocampal place area (PPA), which responds selectively to images of houses, places, and visual scenes; and the extrastriate body area (EBA), which responds specifically to images of bodies and body parts. While the presence of these regions in the mature human brain is well-established, the degree to which children possess these areas and the degree of functional specialization of these areas in children of various ages has thus far remained unclear. This functional magnetic resonance imaging study examined the development of the FFA, EBA, and PPA in healthy, typically developing 7- to 11-year-old children and adults. Our results revealed a right FFA and a bilateral EBA and PPA in the children that were localized in a way consistent with these same regions in adults. In addition, the response profiles of these regions were very similar in adults and children with comparable levels of functional specificity at all of the ages tested. We discuss the implications of this research for understanding abnormal regional specialization for social and nonsocial object categories in individuals with autism spectrum disorders.

Keywords: fMRI, category selectivity, faces, places, bodies

\section{INTRODUCTION}

Prior functional magnetic resonance imaging (fMRI) studies in adult humans have documented the response properties of a small set of brain regions in the extrastriate visual cortex that are remarkably selective in their response to specific categories of social (defined here as containing a representation of another person) or nonsocial (not containing people) visual stimuli. To date, three regions have been identified with striking regularity. One is a face-selective region that has been dubbed the "fusiform face area" (FFA) because it responds more strongly to faces than to any other object category that has been tested. The FFA is localized to the lateral fusiform gyri in the human ventral temporal cortex (e.g., Allison et al., 1994; Puce et al., 1995; Kanwisher et al., 1997; McCarthy et al., 1997). Another region, termed the "extrastriate body area" (EBA), is most responsive to images of bodies and body parts (e.g., Downing et al., 2001). While these first two regions are exquisitely selective for socially relevant stimuli, a third visual processing region, the "parahippocampal place area" (PPA), exhibits selectivity for a class of nonsocial stimuli, images of houses, places, and visual scenes (e.g., Epstein and Kanwisher, 1998; Epstein et al., 1999). While much is known about the localization, organization, and response properties of these categoryselective visual processing regions in adults, relatively little is known about the emergence and development of these regions in children. To date, a handful of functional neuroimaging studies have been reported concerning the FFA and PPA in children, but no studies have addressed the EBA.
The earliest developmental studies to investigate the functional organization of the human extrastriate cortex in children examined the role of the lateral fusiform gyri in face processing. A positron emission tomography study reported areas in the ventral occipitotemporal cortices that were more responsive to faces than to shapes in 2-month-old infants (Tzourio-Mazoyer et al., 2002), suggesting the possibility of very early specialization for faces. However, this study was flawed in its methods, particularly the choice of control stimuli, and thus cannot clearly demonstrate the presence of early face specificity. The results of studies of older children using fMRI and more adequate control stimuli have been much more equivocal. An initial fMRI study of 10- to 12-year olds found bilateral, face-selective activity in the fusiform gyri (Passarotti et al., 2003). In another fMRI study, 5- to 8-year olds failed to exhibit an FFA, but 9- to 11-year olds showed activity in an area of the fusiform gyri similar to the adult FFA (Gathers et al., 2004). Aylward et al. (2005) found that 12- to 14-year-old children but not 8- to 10-year-old children exhibited face-selective activity in a region of the fusiform gyri that was consistent with the expected location of the FFA.

More recently, fMRI studies have continued to examine the development of face-selective activity in the fusiform gyrus in children of various ages. Scherf et al. (2007) compared the activity evoked by pictures of places, faces, and objects in 6- to 8-year-old children, 11- to 14-year-old adolescents, and adults. Replicating some prior findings, they were unable to identify adult-like faceselective regions of activity in the younger children, but they were able to localize the FFA in the adolescents and the adults. In contrast, 
the localization of brain activity evoked by pictures of places and objects was equivalent across the observed age range. Subsequently, Golarai et al. (2007) reported that children as young as 7 years had face-selective activity localized to the fusiform gyri, but the volume of category-selective activation in the FFA and parahippocampal gyrus to faces and places, respectively, increased across the 7- to 11 -year period and correlated with improved recognition memory for the respective stimulus class.

In comparison to the studies of the FFA, studies of children and adolescents have consistently reported the presence of a PPA as early as middle childhood (Golarai et al., 2007; Scherf et al., 2007). However, there are some inconsistencies in the findings regarding the nature of the developmental trajectory thereafter. Scherf et al. (2007) reported bilateral, adult-like PPA activation in 6- to 8-year olds, followed by more right-lateralized (but still bilateral) activation in 11- to 14-year olds, and a return to more evenly bilateral activation in adults. Overall, no difference in the extent of activation among the three age groups was found (Scherf et al., 2007). In contrast, although no age effects on size were found in the right PPA, Golarai et al. (2007) found that the left PPA was significantly smaller in children (ages 7-11) than in adults. Adolescents (ages 12-16 years) also showed a trend towards smaller size than adults. The three-fold increase in size of the left PPA across the observed age range was suggested to reflect the increase in selectivity reported (Golarai et al., 2007).

As the summaries above illustrate, prior studies have generated initial, often conflicting findings. Consequently, it is difficult to arrive at a clear conclusion regarding the development of the PPA and FFA, and there are no available developmental findings for the EBA. Thus, the prior work serves to highlight the need for additional developmental data. In the present study, we wished to further assess the development of the FFA and PPA in children in order to potentially clarify the controversies over their presence or absence in children. We also sought to conduct an initial fMRI study of the EBA in children. To this end, we employed a stimulus set that included faces, bodies, and places, along with flowers and sporting goods, in a blocked-design fMRI study of adults and children ages 7-11 years.

This kind of developmental study is critical for translational efforts. Studies of people with autism spectrum disorders have indicated abnormal development of face-selective responses in the FFA (for a review, see Schultz, 2005). However, in the absence of a better understanding of the normative developmental trajectory in this region, it is difficult to evaluate whether this abnormality plays a causal role in the emergence of the disorder or is an effect of the lack of interest in faces that is a characteristic of autism spectrum disorders.

\section{MATERIALS AND METHODS PARTICIPANTS}

Ten adults (age range $=18.9-29.2$ years; $M=22.5, \mathrm{SD}=3.5$ years; seven females) and 22 children (age range $=7.2-11.9$ years; $M=9.7$ years, $\mathrm{SD}=1.3$ years; 14 females) participated in this study. Participants had no history of neurological or psychiatric disorders as measured by self (adults) or parent (for the children) report and had normal or corrected-to-normal vision. All participants were right-handed as assessed by the Edinburgh Handedness Inventory (Oldfield, 1970). Participants (adults) or their parents (for the children) provided written consent for this study, which was approved by the local Human Investigations Committee.

\section{PROCEDURE}

\section{Stimuli and design}

Figure 1 shows sample stimuli for each of the five categories of visual stimuli used in this study. In a block design, stimulus types were shown in 12-s stimulation blocks alternating with 12-s fixation blocks. No single type of stimulation block was shown twice in a row. Within each stimulus block, 24 images from that category were displayed for $500 \mathrm{~ms}$ each. In order to ensure that participants were attending, they were instructed to push a button when the same image was presented twice in immediate succession, which occurred approximately three times per run. Identical stimuli were used for child and adult participants. The study involved two scanning runs. The first run lasted $6.23 \mathrm{~min}$ and included five blocks each of photographs of faces, flowers, and bodies. The second run was 4.23 min long and consisted of five blocks each of photographs of places and sporting goods.

\section{Imaging}

Scanning was performed on a 3-Tesla Signa Excite HD scanner system with $50-\mathrm{mT} / \mathrm{m}$ gradients from General Electric Health Technologies (General Electric, Waukesha, WI). Parallel imaging was performed using an eight-channel head coil. Participants' heads were immobilized using tape and a vacuum cushion. Sixty-eight high-resolution anatomical images were acquired using a $3 \mathrm{D}$ fast SPGR pulse sequence $(\mathrm{TR}=500 \mathrm{~ms} ; \mathrm{TE}=20 \mathrm{~ms} ; \mathrm{FOV}=24 \mathrm{~cm}$; image matrix $=256^{2}$; voxel size $\left.=0.9375 \times 0.9375 \times 1.9 \mathrm{~mm}\right)$ and used for coregistration with the functional data. These structural images were aligned in the near-axial plane defined by the anterior and posterior commissures. Whole-brain functional images were acquired using an echoplanar imaging sequence sensitive to blood oxygenation level dependent contrast $(\mathrm{TR}=2000 \mathrm{~ms}$; $\mathrm{TE}=35 \mathrm{~ms}$; FOV $=24 \mathrm{~cm}$; image matrix $=64^{2}$; voxel size $3.75 \mathrm{~mm} \times 3.75 \mathrm{~mm}$ $\times 3.8 \mathrm{~mm}$; 34 axial slices). Functional and structural images were coplanar. Global field homogeneity was ensured through use of a semi-automated high-order shimming program, and the first four radio frequency excitations of each run were discarded in order to have steady state equilibrium.

\section{Analyses}

Image preprocessing was performed with custom programs and SPM modules (Wellcome Department of Cognitive Neurology, UK). Head motion was detected by center-of-mass measurements. None of the 22 children and 10 adults included in the data set described in this paper exhibited greater than a $3-\mathrm{mm}$ deviation in the center of mass in any dimension. However, we did exclude two additional adults and seven additional children because they exhibited excessive head motion. Images were time-adjusted to compensate for the interleaved slice acquisition and then motion-corrected to compensate for small $(<3 \mathrm{~mm})$ head movements. Our analyses employed a voxel-based analytical approach. The realigned and motion-corrected images were first normalized to the Montréal Neurological Institute (MNI) template found in SPM 99. These normalized functional data were then high-pass filtered and spatially smoothed with a 6-mm isotropic Gaussian kernel prior to statistical analysis. These realigned, motion-corrected, normalized, and smoothed data were used in the random-effects analyses described below. By normalizing the imaging data from adults and children 

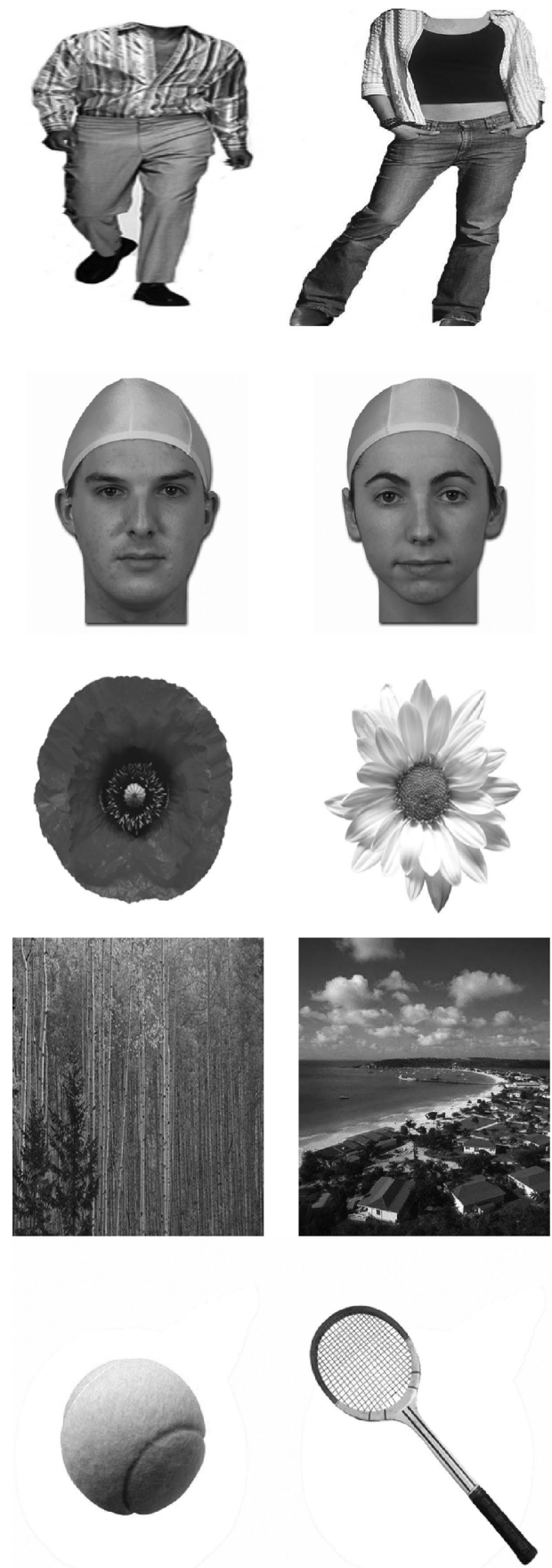
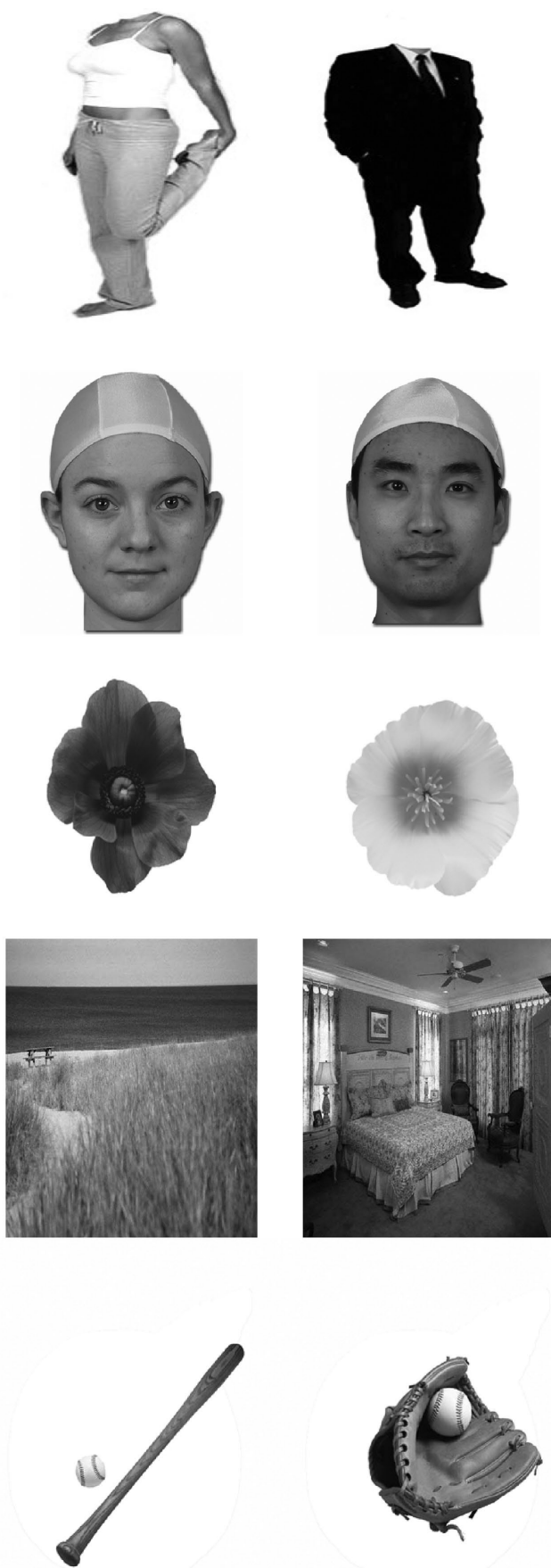

FIGURE 1 | Sample stimuli. The stimuli for this study included black-and-white images of bodies (Row 1), faces (Row 2), flowers (Row 3), places (Row 4), and sporting goods (Row 5).

to the MNI space, we were able to compare functional activation foci from both groups of participants within a common template. Kang et al. (2002) provided an empirical validation of normalization for analysis of fMRI data from children. They found very small differences (relative to the resolution of fMRI data) in the spatial correspondence among several brain loci between young children and adults after warping child and adult fMRI data into a common space. These and other similar findings (Burgund et al., 2002) gave us confidence in the feasibility of directly comparing data from adults and children in a common, adult space.

In order to localize the three functionally defined regions that were of primary interest in this study (the FFA, PPA, and EBA), randomeffects assessments of the differences between key pairs of conditions at the expected peak of the hemodynamic response (HDR) were performed. These analyses consisted of the following steps: (1) The epochs of image volumes beginning one image before $(-2.0 \mathrm{~s})$ and 
11 images after (22s) the onset of each block containing pictures of a particular stimulus category of interest were extracted from the continuous time series of volumes and averaged together by stimulus type. (2) The average intensity of the HDR within its expected peak (6-16-s post stimulus onset) was computed. (3) A $t$-statistic was then computed at each voxel within the brain to quantify the HDR differences between sets of two conditions of interest (i.e., FFA $=$ faces versus flowers; $\mathrm{EBA}=$ bodies versus flowers; $\mathrm{PPA}=$ places versus sporting goods). This process was performed separately for each subject. (4) The individual $t$-maps created in the preceding step were then subjected to a random-effects analysis that assessed the significance of stimulus-category response differences across subjects. This procedure was preformed separately for children and adults.

\section{RESULTS}

We organize our results first by brain region (the FFA, PPA, and EBA). Then, within each brain region, the findings are organized around a set of key questions. Specifically, by comparing the locations of activations, we evaluated whether children exhibit adultlike FFAs, PPAs, and EBAs. Then, by examining the response properties of the localized FFAs, EBAs, and PPAs, we evaluated whether these regions differed in children of various ages and in children as compared to adults with regard to their selectivity for the defining stimulus categories.

\section{FUSIFORM FACE AREA}

Do children exhibit an adult-like FFA by middle childhood? Consistent with a prior fMRI study of adults (McCarthy et al., 1997), the FFA was defined as a cluster of contiguous voxels in and around the fusiform gyri that exhibited a significantly greater response to faces than to flowers $(p<0.05)$. Flowers were chosen for this comparison because they are a category of object with a moderate degree of visual complexity, and individual species of flowers can be identified at the subordinate level by comparison of different blossoms. The locations of the adult and child FFAs are illustrated in the leftmost panel of Figure 2. As can be seen, in adults (red color map), a region of face-related activation was localized to the lateral fusiform gyrus in both the right (47x, $-51 y,-14 z)$ and left hemispheres $(-37 x,-53 y,-17 z)$. In adults, the FFA was larger in the right hemisphere (175 voxels) than in the left hemisphere ( 45 voxels). In children (blue color map), an FFA was identified only in the right hemisphere fusiform gyrus (MNI coordinates: $41 x,-44 y .-17 z$; voxel count $=108)$. The centroid of the adult right-hemisphere FFA localized in this study was localized slightly more lateral $(6 \mathrm{~mm})$, posterior $(7 \mathrm{~mm})$, and inferior $(3 \mathrm{~mm})$ compared to the child FFA. The stereotaxic coordinates reported for the adult and child FFAs observed in this study are consistent with those from many other published studies of face perception using a blocked stimulus design (e.g., Clark et al., 1996: 37x, $-55 y,-10 z$; Haxby et al., 1994: 38x, $-58 y$, 0z; Kanwisher et al., 1997: 40x,-55y,-10z; Clark et al., 1998: 39x, $-56 y,-22 z$; Gauthier et al., 1999: 41x,-51y,-12z; McCarthy et al., 1997: 40x, $-59 y,-22 z$; Sergent et al., 1992: $37 x,-55 y,-11 z$; average of these seven studies: $39 x,-56 y,-13 z)$. Note the coordinates from the average of these seven prior studies are within the spatial extent of the current study's activations. The waveforms from the right-hemisphere child and adult FFAs are illustrated in the leftmost panels of Figure 3. As expected, in both groups of subjects, the response to faces was significantly greater than the response to flowers. Also note that inspection of these plots reveals that the absolute magnitude of responses to faces and flowers was quite similar for children and adults in the FFA. Likewise, the magnitude of the difference between the responses to faces and flowers was similar.

Although the FFAs identified here in adults and children exhibited a greater response to faces than to flowers [(children: $t=2.80$, $p=0.01$ ); (adults: $t=2.88, p=0.01$ )], we found no significant difference between the responses to faces and bodies (presented in the same run $)$ in these same regions in children $(t=0.11, p=0.92)$ and adults $(t=0.40, p=0.70)$. This is likely indicative of the presence of the putative "fusiform body area" (Peelen and Downing, 2005). This region is distinct from but adjacent to the FFA (Schwarzlose et al., 2005); however, we were not able to make this fine-grained distinction here in the absence of high-resolution fMRI data. These results do suggest the presence of a fusiform body area in children, an issue that we intend to address in future research.

Do the response properties of the FFA change over middle childhood or between middle childhood and adulthood? To evaluate this question, we calculated a face selectivity index for each participant using the following formula: $\mathrm{SI}_{\text {faces }}=($ mean response $\mathrm{faces}-$ mean response $\left._{\text {places }}\right) \times 100$. Children $\left(\right.$ mean $\left.\mathrm{SI}_{\text {faces }}=0.14 \% ; \mathrm{SE}=0.05 \%\right)$ and adults (mean $\mathrm{SI}_{\text {faces }}=0.20 \% ; \mathrm{SE}=0.07 \%$ ) did not significantly differ in the average selectivity of their right-hemisphere FFAs $(F=0.43, p=0.52)$. Furthermore, no correlation was found between the selectivity of the right FFA for faces and age in children $(r=0.10, p=0.65)$. While we defined the FFA in children and adults by comparing responses to faces and flowers, we chose a different category (places) to calculate the selectivity index for this region. This helped us to circumvent issues of non-independence (e.g., Vul and Kanwisher, in press) in our analyses. That is, by employing a third object category that was not used to define the FFA, we were more conservative in allowing for the possibility that the response could be equivalent to faces and places. We also examined the question of differences in selectivity by calculating this index using faces and flowers. The results were essentially identical. This same strategy was used for the analyses of the EBA and PPA.

\section{EXTRASTRIATE BODY AREA}

Do children exhibit an adult-like EBA by middle childhood? The EBA was defined as a contiguous cluster of body-selective voxels (relative to pictures of faces) in the lateral occipitotemporal cortex (Downing et al., 2001). As illustrated in the middle panel of Figure 2, bilateral EBA activation was identified through randomeffects analyses in both children and adults $(p<0.01)$. In children, the EBA was larger in the right hemisphere $(45 x,-66 y, 9 z, 712$ voxels than in the left hemisphere $(-39 x,-67 y, 9 z, 251$ voxels). The opposite pattern was observed in adults, wherein the left EBA $(-39 x,-79 y, 17 z, 480$ voxels) was larger than the right-hemisphere EBA $(39 x,-83 y, 15 z, 240$ voxels). The coordinates for the adult and child EBAs were very similar to each other and consistent with the coordinates originally reported by Downing et al. (2001): 51x, $-71 y, 1 z$ and $-51 x,-72 y, 8 z$, as well as other published descriptions of the EBA (e.g., Astafiev et al., 2004; $-40 x,-58 y, 7 z ;-48 x,-69 y$, 


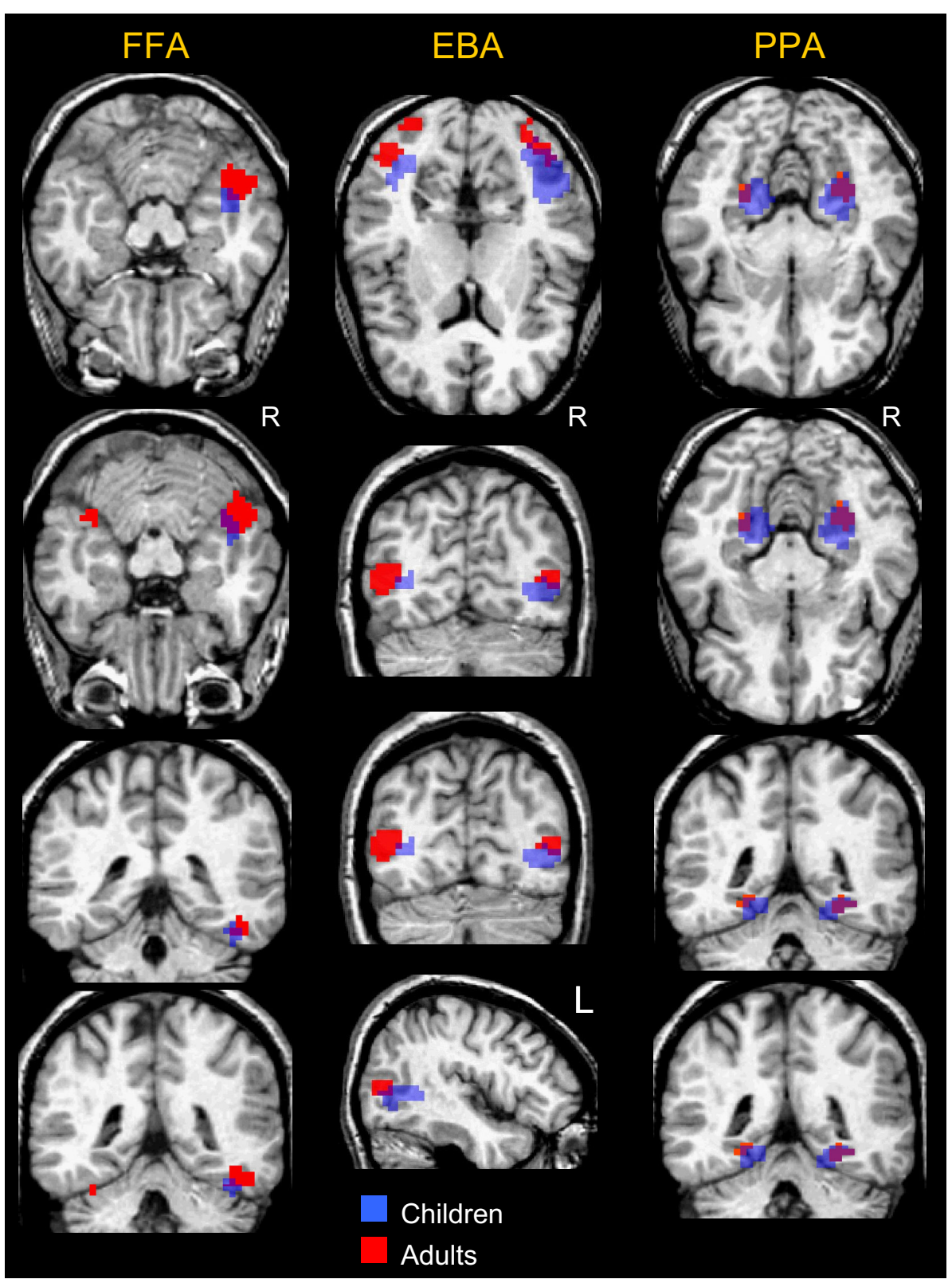

FIGURE 2 |Activation maps. The results of statistical comparisons used to identify category-selective brain regions are presented for children (in blue) and adults (in red). The first column contains images of the identified FFA. The second column includes activation maps for the EBA. The third column shows the PPA.

$-6 z$; Taylor et al., 2007: $-47 x,-71 y$ and $0 z ; 49 x,-65 y, 4 z)$. The coordinates from these papers are well within the spatial extent of the current activations. The waveforms from the right and left child and adult EBAs are illustrated in the rightmost panels of Figure 3. In both groups of subjects, the response to pictures of bodies was significantly greater than the response to faces or flowers.

Do the response properties of the EBA change over middle childhood or between middle childhood and adulthood? We calculated a body selectivity index for each participant using the following formula: $\mathrm{SI}_{\text {bodies }}=\left(\right.$ mean response ${ }_{\text {bodies }}-$ mean response flowers $) \times 100$. Children (mean $\mathrm{SI}_{\text {bodies }}=0.30 \% ; \mathrm{SE}=0.06 \%$ ) and adults (mean $\mathrm{SI}_{\text {bodies }}=0.31 \% ; \mathrm{SE}=0.11 \%$ ) did not differ in the average selectivity of their right-hemisphere EBAs $(F=0.04, p=0.85)$. Likewise, children $\left(\right.$ mean $\left.\mathrm{SI}_{\text {bodies }}=0.24 \% ; \mathrm{SE}=0.06 \%\right)$ and adults $\left(\right.$ mean $\mathrm{SI}_{\text {bodies }}=0.28 \%$; $\mathrm{SE}=0.05 \%)$ did not differ in the average selectivity of their left-hemisphere EBAs $(F=0.23, p=0.64)$. Furthermore, no correlation was found between the selectivity of the right $(r=-0.21, p=0.35)$ or left $(r=-0.24, p=0.29)$ EBA for bodies and age in children.

\section{PARAHIPPOCAMPAL PLACE AREA}

Do children exhibit an adult-like PPA by middle childhood? The PPA was defined as a cluster of contiguous voxels in and around the parahippocampal gyri that exhibited a significantly greater response to pictures of places than to pictures of sporting goods 

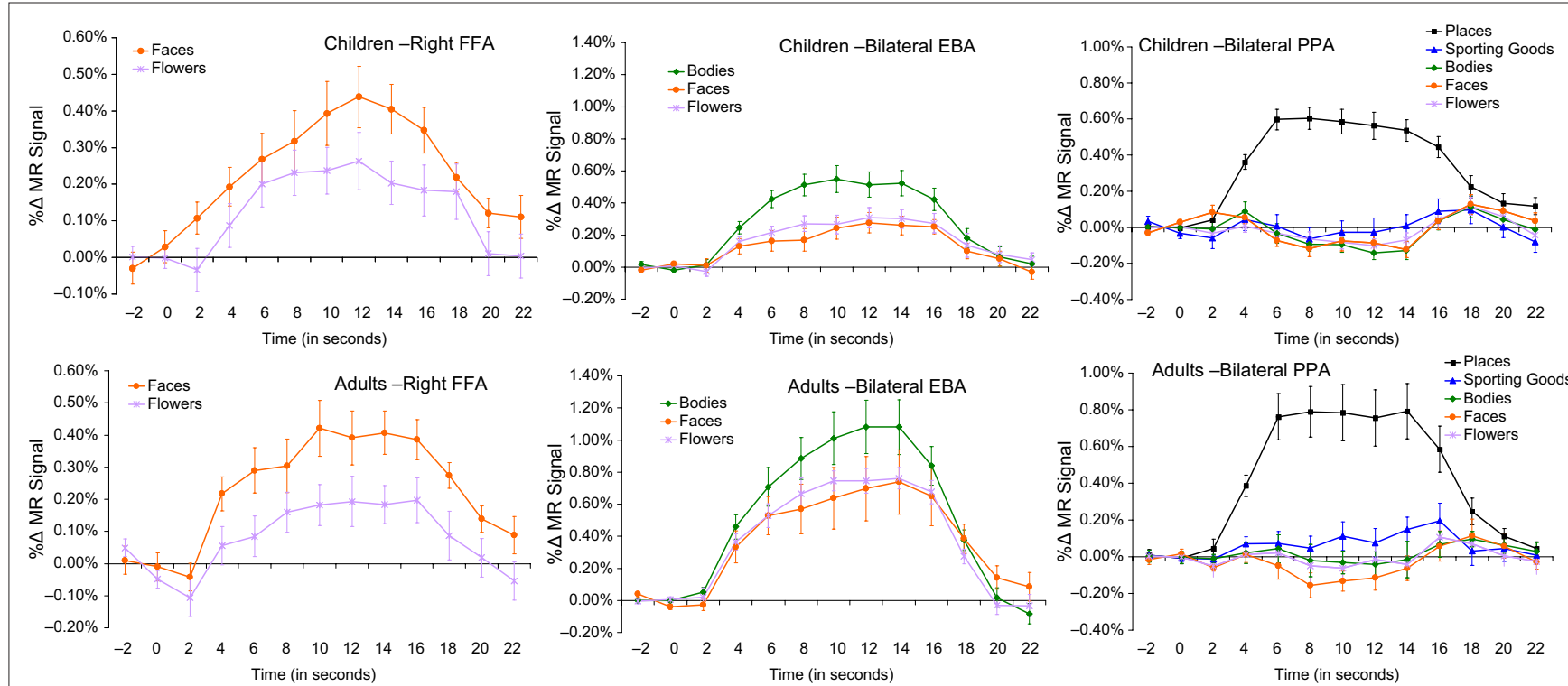

FIGURE 3 |Waveforms. In the first column are the HDR waveforms from the identified FFA in children (top) and adults (bottom) for faces (orange squares) versus flowers (purple Xs). The second column contains waveforms from the EBA for children (top) and adults (bottom) for bodies (green diamonds) relative to faces (orange squares) and flowers (purple Xs). Waveforms from the PPA in children (top) and adults (bottom) are presented in the third column, with data from places (black rectangles) presented relative to sporting goods (blue triangles), bodies (green diamonds), faces (orange squares), and flowers (purple Xs). $(p<0.0001)$. Our analyses revealed a place-selective region in the bilateral parahippocampal gyri of adults and children. The locations of the adult and child PPAs are illustrated in the rightmost panel of Figure 2. As can be seen, in children and adults, a region of place-selective activation was localized to the parahippocampal gyrus in both the right and left hemispheres. In both children and adults, the PPA was larger in the right hemisphere (children: $25 x,-43 y,-10 z, 516$ voxels; adults: $28 x,-47 y,-3 z, 104$ voxels) than in the left hemisphere (children: $-19 x,-43 y,-5 z$, 427 voxels; adults: $-25 x,-49 y,-4 z, 40$ voxels). The coordinates for the adult and child PPAs were very similar to each other and to previously reported coordinates from studies of adults (e.g., Epstein et al., 2003: experiment $1=28 x,-46 y,-10 z ;-26 x,-48 y$, $-9 z$; experiment $2=29 x,-43 y,-11 z ;-28 x,-47 y,-10 z)$. The waveforms from the right and left child and adult PPAs are illustrated in the rightmost panels of Figure 3. In both groups of subjects, the response to pictures of places was significantly greater than the response to any of the other categories of stimuli viewed by the participants.

Do the response properties of the PPA change over middle childhood or between middle childhood and adulthood? Here, we calculated a place selectivity index for each participant using the following formula: $\mathrm{SI}_{\text {places }}=$ (mean response ${ }_{\text {places }}-$ mean respons$\left.\mathrm{e}_{\text {sporting goods }}\right) \times 100$. Children (mean $\left.\mathrm{SI}_{\text {places }}=0.57 \% ; \mathrm{SE}=0.06 \%\right)$ and adults (mean $\left.\mathrm{SI}_{\text {places }}=0.67 \% ; \mathrm{SE}=0.07 \%\right)$ did not differ in the average selectivity of their right-hemisphere PPAs $(F=1.00, p=0.33)$. Likewise, children (mean $\mathrm{SI}_{\text {places }}=0.54 \%$; $\mathrm{SE}=0.06 \%$ ) and adults (mean $\mathrm{SI}_{\text {places }}=0.59 \% ; \mathrm{SE}=0.10 \%$ ) did not differ in the average selectivity of their left-hemisphere PPAs $(F=0.30, p=0.59)$. Furthermore, no correlation was found between the selectivity for places of the right $(r=-0.17, p=0.44)$ or left $(r=-0.28, p=0.21)$ PPA and age in children.

\section{DISCUSSION}

In this study, we sought to clarify prior, sometimes conflicting, findings regarding the presence or absence of an FFA and a PPA in school-aged children. Additionally, we sought to provide a first investigation of the EBA in children. To do this, we conducted an fMRI study to examine the developmental trajectories of these three social and nonsocial category-selective extrastriate visual regions in terms of their localization and specificity of responses in 7- to 11-year-old children. We also studied a sample of adults in order to provide an anchoring assessment of the mature state of these category-selective regions.

We identified a face-selective region of the right fusiform gyrus in 7- to 11-year-old children and adults. The right FFA was localized similarly in adults and children and the selectivity of this region did not vary as a function of age. These findings contrast with two prior fMRI studies that failed to identify the FFA in a group of children within this age range (Aylward et al., 2005; Scherf et al., 2007), but are consistent with three other studies that have demonstrated the existence of an FFA in school-age children (e.g., Passarotti et al., 2003; Gathers et al., 2004; Golarai et al., 2007). A prior report described a potential shift in the localization of the FFA in children versus adolescents and adults. Scherf et al. (2007) reported that children exhibited a more posterior and lateral face-sensitive region of the right fusiform gyrus; however, with a more generous contrast, they instead reported two different regions that were more dorsal and medial to the adult FFA. In contrast, we found that the FFA in children was slightly more anterior and medial to the adult FFA. It is difficult to evaluate the importance of the reported shifts in localization. In both fMRI studies, normalization of the fMRI data was used to allow direct comparisons of participants of various ages. However, different normalization algorithms were employed between the two studies. Within either study, the differences in 
localization could be attributable to the normalization process. Further research is necessary to determine whether these reported shifts are indeed meaningful.

It does appear potentially important that the child FFA was only found in the right hemisphere, whereas the adult participants displayed an FFA in both hemispheres. Similar right-lateralization of the FFA in children followed by bilateral adult activity has been reported by other researchers (Gathers et al., 2004; Aylward et al., 2005). Future research should address the behavioral consequences of the emergence of a left FFA during childhood. Behavioral studies have reported a variety of developmental changes in different aspects of face processing across the 7-to 11-year period, including a shift from more feature-based to configuration-based strategies (e.g., Carey and Diamond, 1977; Diamond and Carey, 1977; Carey, 1992), improvements in emotion discrimination (Camras and Allison, 1985; Gnepp, 1989; Felleman et al., 1993), the emergence of an inversion effect (e.g., Carey and Diamond, 1977; Carey, 1992), and improvements in recognition abilities (Baenninger, 1994; Chung and Thomson, 1995; Brace et al., 2001; Taylor et al., 2004; for review, see Passarotti et al., 2007). It could be that the neurofunctional change observed here would be related to these behavioral changes.

Notably, we did not observe any change in the response properties (defined here as the specificity of the response for the preferred stimulus class compared to another category of stimuli) of the right FFA across this age range. This finding supports the conclusion that the right FFA is already "adult-like" by age 7 years in terms of differentiating faces and objects. This finding is consistent with the results of comparisons of functional characteristics of the FFA in 9- to 11-year-old children reported by Gathers et al. (2004). However, this finding differs from the findings of Aylward et al. (2005) that suggested that children lack a face-selective region in the fusiform gyrus.

This study is the first to localize the EBA in children. This region was identified bilaterally with very similar coordinates in children and adults. In both groups, the coordinates were similar to those reported in prior studies of adults (Downing et al., 2001; Astafiev et al., 2004; Taylor et al., 2007). The selectivity for images of bodies did not differ between children and adults, and there was no correlation between age and specificity in the children. These findings demonstrate, for the first time, the presence of the EBA in children. Furthermore, they illustrate that the EBA is already adult-like in terms of localization and specificity by 7 years of age.

The PPA was localized in both children and adults in the bilateral parahippocampal gyri, consistent with previous reports (Passarotti et al., 2003; Golarai et al., 2007; Scherf et al., 2007). Moreover, the coordinates for the regions identified in the two groups were similar to each other and to previous reports (e.g., Epstein and Kanwisher, 1998). Notably, we found that adults and children showed more place-sensitive voxels in the right hemisphere than in the left hemisphere, in accord with previous adult findings (Epstein and Kanwisher, 1998; Passarotti et al., 2003). This similarity contrasts with the findings reported by Scherf et al. (2007) that suggested that young children showed bilateral activity, older children illustrated right-lateralized activity, and adults demonstrated more evenly bilateral activation patterns again, although they noted that there was a significantly larger proportion of active voxels in the right than the left hemisphere in all age groups. As with the EBA, no age differences were found in selectivity of this area, in contrast with other findings (Golarai et al., 2007) that suggested that children had lower place selectivity than adults in the left hemisphere.

While we believe that this study provides a number of important findings to the growing literature on the development of category-selective regions in the human brain, the study suffers from a number of limitations that should be addressed in future work. For example, scanning younger children would likely be more informative with regard to informing competing theories of neurofunctional brain development. Moreover, our design was cross-sectional. A longitudinal design would have allowed us to examine the shape of developmental trajectories of brain functioning in the different, category-selective regions, and this would have provided more detail on the nature of differences in development pathways among these brain regions than did comparisons of different children at various ages and comparisons between children and adults. Lastly, future work should include more categories of images. In particular, the addition of classes of stimuli such as letters and numbers potentially could result in longer and/or later developmental trajectories owing to later learning and familiarity with such stimuli during childhood.

Along with these potentially later-developing categories, it would be interesting to explore in greater detail some visual categories that draw upon multiple brain regions for their processing. For example, examinations of complex facial stimuli, possibly displaying emotions, motion, or different forms of eye contact, could draw upon the FFA, the posterior superior temporal sulcus region (STS), and the amygdala. Currently, it is known that the STS has relatively mature functioning for some tasks during middle childhood, such as assessing the intentionality of eye movements (Mosconi et al., 2005), but it is still developing for other processes, including the perception of biological motion (Carter and Pelphrey, 2006). This could signify that different networks of regions that include the posterior STS are developing at different rates owing to different interactions, resulting in varying degrees of tuning for stimuli.

Our findings raise important questions about differences in the developmental trajectories of the PPA, EBA, and FFA. In particular, we and others have found that the PPA is the most adult-like at a young age out of the three regions examined. Why should this be so? We hypothesize that this difference in developmental trajectories can be explained by the possibility of fewer regions involved in place processing more generally, resulting in fewer interactions that need to develop and relatively complete development early in ontogeny, along with less potential for complexity inherent in these stimuli relative to faces and bodies. Faces and bodies are social entities that not only can be viewed from many different angles, but can convey identity through simple features, motion patterns, and feature configurations. They can also illustrate various emotions and other conditions without changing their identities. Moreover, all of these factors can and do change rapidly, and small nuances are important in social interactions. Also, whereas a viewer can change his or her location relative to a place and therefore change his or her viewpoint, it is highly unlikely that the location will change relative to him while he stays completely still. Individuals do not move quickly relative to places that they are viewing, resulting in 
lesser demands on processing speed as compared to watching other individuals who move unexpectedly and with great speed. Viewers are not in control of their perspectives on the faces and bodies of others, as other people are separate agents. All of these factors might increase the perceptual complexity of social stimuli like faces and bodies and the amount of attention that needs to be allocated to these categories of stimuli for proper processing.

Finally, our findings might offer important implications for understanding the etiology of social deficits in autism spectrum disorders. Previous reports have identified abnormalities in the localization and specificity of the FFA in adults with autism spectrum disorders (e.g., Pierce et al., 2001; Hubl et al., 2003; Schultz et al., 2003; Ashwin et al., 2007; Pelphrey et al., 2007). Understanding the normative developmental pathway and mechanisms by which this region typically develops could provide invaluable data for understanding the causes of FFA dysfunction in individuals with autism spectrum disorders. This information could also inform our understanding of treatments for social perception deficits in

\section{REFERENCES}

Allison, T., Ginter, H., McCarthy, G., Nobre, A. C., Puce, A., Luby, M., and Spencer, D. (1994). Face recognition in human extrastriate cortex. J. Neurophysiol. 71, 821-825.

Ashwin, C., Baron-Cohen, S., Wheelwright, S., O'Riordan, M., and Bullmore, E. T. (2007). Differential activation of the amygdala and the 'social brain' during fearful face processing in Asperger Syndrome. Neuropsychologia 45, 2-14.

Astafiev, S. V., Stanley, C. M., Shulman, G. L., and Corbetta, M. (2004). Extrastriate body area in human occipital cortex responds to the performance of motor actions. Nat. Neurosci. 7, 542-548.

Aylward, E. H., Park, J. E., Field, K. M., Parsons, A. C., Richards, T. L., Cramer, S. C., and Meltzoff, A. N. (2005). Brain activation during face perception: evidence of a developmental change. J. Cogn. Neurosci. 17, 308-319.

Baenninger, M. (1994). The development of face recognition: featural or configurational processing? J. Exp. Child Psychol. 57, 377-396.

Brace, N. A., Hole, G. J., Kemp, R. I., Pike, G. E., Van Duuren, M., and Norgate, L. (2001). Developmental changes in the effect of inversion: using a picture book to investigate face recognition. Perception 30, 85-94.

Burgund, E. D., Kang, H. C., Kelly, J. E., Buckner, R. L., Snyder, A.Z., Petersen, S. E., et al. (2002). The feasibility of a common stereotactic space for children and adults in fMRI studies of development. Neuroimage 17, 184-200.

Camras, L., and Allison, K. (1985). Children's understanding of emotional facial expressions and verbal labels. $J$. Nonverbal Behav. 9, 84-94.

Carey, S. (1992). Becoming a face expert. Philos. Trans. R. Soc. Lond., B, Biol. Sci. 335, 95-102.

Carey, S., and Diamond, R. (1977). From piecemeal to configurational representation of faces. Science 195, 312-314.

Carter, E. J., and Pelphrey, K. A. (2006). School-aged children exhibit domainspecific responses to biological motion. Soc. Neurosci. 1, 396-411.

Chung, M.S., and Thomson, D. M. (1995). Development of face recognition. $\mathrm{Br}$. J. Psychol. 86, 55-87.

Clark, V. P., Keil, K., Maisog, J. M. Courtney, S., Ungerleider, L. G., and Haxby, J. V. (1996). Functional magnetic resonance imaging of human visual cortex during face matching: a comparison with positron emission tomography. Neuroimage 4, 1-15.

Clark, V. P., Maisog, J. M., and Haxby, J. V. (1998). fMRI study of face perception and memory using random stimulus sequences. J. Neurophysiol. 79 , 3257-3265.

Diamond, R., and Carey, S. (1977). Developmental changes in the representation of faces. J. Exp. Child Psychol. 23, 1-22.

Downing, P. E., Jiang, Y., Shuman, M., and Kanwisher, N. (2001). A cortical area selective for visual processing of the human body. Science 293, 2470-2473.

Epstein, R., Graham, K. S., and Downing, P. E. (2003). Viewpointspecific scene representations in human parahippocampal cortex. Neuron 37, 865-876.

Epstein, R., Harris, A., Stanley, D., and Kanwisher, N. (1999). The parahippocampal place area:

autism with the goal of normalizing developmental pathways or supporting alternative, compensatory mechanisms.

In conclusion, we have identified face-, body-, and place-sensitive areas of the cortex in school-aged children that are somewhat, but not completely, similar to those of adults. Importantly, it further elucidates the developmental trajectories for these category-specific regions. This work also has import for research on autism spectrum disorders. Future work should study these regions longitudinally, along with other category-selective regions identified in adults.

\section{ACKNOWLEDGMENTS}

Kevin Pelphrey was supported by an NIMH Career Development Award (K01 MH071284) and by the John Merck Scholars Fund. James Morris was supported by an NRSA from the NIMH. This research was supported by the John Merck Scholars Fund, NIMH grant MH071284, and Autism Speaks. This paper resulted from Juliana Lopez's honors thesis, completed when she was an undergraduate at Duke University.

recognition, navigation, or encoding? Neuron 23, 115-125.

Epstein, R., and Kanwisher, N. (1998) A cortical representation of the local visual environment. Nature 392, 598-601.

Felleman,E.S.,Barden, R.C., Carlson, C. R., Rosenberg, L., and Masters, J.C. (1993). Children's and adults' recognition of spontaneous and posed emotional expressions in young children. Dev Psychol. 19, 405-413.

Gathers, A. D., Bhatt, R., Corbly, C. R. Farley, A. B., and Joseph, J. E. (2004). Developmental shifts in cortical loci for face and object recognition. Neuroreport 15, 1549-1553.

Gauthier, I., Tarr, M. J., Anderson, A. W. Skudlarski, P., and Gore, J. C. (1999) Activation of the middle fusiform 'face area' increases with expertise in recognizing novel objects. Nat. Neurosci. 2, 568-573.

Gnepp, J. (1989). Children's use of personal information to understand other people's feelings. In Children's Understanding of Emotion, C. Saarni and P. L. Harris, eds (New York, NY, Cambridge University Press), pp. 3-24.

Golarai, G., Ghahremani, D. G., WhitfieldGabrieli, S., Reiss, A., Eberhardt, J. L., Gabrieli, J. D., and Grill-Spector, K (2007). Differential development of high-level visual cortex correlates with category-specific recognition memory. Nat. Neurosci. 10, 512-522.

Haxby, J.V., Horwitz, B., Ungerleider, L. G., Maisog, J. M., Pietrini, P., and Grady, C. L. (1994). The functional organization of human extrastriate cortex: a PET-rCBF study of selective attention to faces and locations. J. Neurosci. 14, 6336-6353.
Hubl, D., Bèolte, S., Feineis-Matthews, S., Lanfermann, H., Federspiel, A., Strik, W., Poustka, F., and Dierks, T. (2003). Functional imbalance of visual pathways indicates alternative face processing strategies in autism. Neurology 61, 1232-1237.

Kang, H.C., Burgund, E. D., Lugar, H. M., Petersen, S. E., and Schlaggar, B. L. (2002). Comparison of functional activation foci in children and adults using a common stereotactic space. Neuroimage 19, 16-28.

Kanwisher, N., McDermott, J., and Chun, M.M.(1997). The fusiform face area: a module in human extrastriate cortex specialized for face perception. J. Neurosci. 17, 4302-4311.

McCarthy, G., Puce, A., Gore, J. C., and Allison, T. (1997). Face-specific processing in the human fusiform gyrus. J. Cogn. Neurosci. 9, 605-610.

Mosconi, M.W., Mack, P.B., McCarthy, G., and Pelphrey, K. A. (2005). Taking an "intentional stance" on eye-gaze shifts: a functional neuroimaging study of social perception in children. Neuroimage 27, 247-252.

Oldfield, R.C. (1971). The assessment and analysis of handedness: the Edinburgh inventory. Neuropsychologia, 9, 97-113.

Passarotti, A. M., Paul, B. M., Bussiere, J. R., Buxton, R. B., Wong, E. C., and Stiles, J. (2003). The development of face and location processing; an fMRI study. Dev. Sci. 6, 100-117.

Passarotti, A. M., Smith, J., DeLano, M., and Huang, J. (2007). Developmental differences in the neural bases of the face inversion effect show progressive tuning of face-selective regions to the upright orientation. Neuroimage 34, 1708-1722. 
Peelen, M. V., and Downing, P. E. (2005). Selectivity for the human body in the fusiform gyrus. J. Neurophysiol. 93, 603-608.

Pelphrey, K. A., Morris, J. P., McCarthy, G., and LaBar, K. S. (2007). Perception of dynamic changes in facial affect and identity in autism. Soc. Cogn. Affect. Neurosci. 2, 140-149.

Pierce, K., Mueller, R. A., Ambrose, J., Allen, G., and Courchesne, E. (2001). Face processing occurs outside the fusiform 'face area' in autism: evidence from functional MRI. Brain 124, 2059-2073.

Puce, A., Allison, T., Gore, J. C., and McCarthy, G. (1995). Face-sensitive regions in human extrastriate cortex studied by functional MRI. J. Neurophysiol. 74, 1192-1199.

Scherf,K.S.,Behrmann,M.,Humphreys, K., and Luna, B. (2007). Visual categoryselectivity for faces, places and objects emerges along different developmental trajectories. Dev. Sci. 10, 15-30.

Schultz, R. T. (2005). Developmental deficits in social perception in autism: the role of the amygdala and fusiform face area. Int. J. Dev. Neurosci. 23, 125-141.

Schultz, R. T., Grelotti, D. J., Klin, A., Kleinman, J., Van der Gaag, C., Marois, R. and Skudlarski, P. (2003). The role of the fusiform face area in social cognition: implications for the pathobiology of autism. Philos. Trans. R. Soc. B. 358, 415-427.

Schwarzlose, R. F., Baker, C. I., and Kanwisher, N. (2005). Separate face and body selectivity on the fusiform gyrus. J. Neurosci. 25, 11055-11059.

Sergent, J., Ohta, S., and MacDonald, B. (1992). Functional neuroanatomy of face and object processing. A positron emission tomography study. Brain 115, 15-36.
Taylor,J.C., Wiggett,A. J., and Downing, P. E. (2007). Functional MRI analysis of body and body part representations in the extrastriate and fusiform body areas. J. Neurophysiol. 98, 1626-1633.

Taylor, M.J., Batty, M., and Itier, R. J. (2004). The faces of development: a review of early face processing over childhood. J. Cogn. Neurosci. 16, 1426-1442.

Tzourio-Mazoyer, N., De Schonen, S. Crivello, F., Reutter, B., Aujard, Y., and Mazoyer, B. (2002). Neural correlates of woman face processing by 2-month-old infants. Neuroimage 15, 454-461.

Vul, E., and Kanwisher, N. (in press). Begging the question: the nonindependence error in fMRI data analysis. In Foundations and Philosophy for Neuroimaging, S. Hanson, and M. Bunzl, eds.

Conflict of Interest Statement: The authors declare that the research was conducted in the absence of any commercial or financial relationships that could be construed as a potential conflict of interest.

Received: 28 July 2009; paper pending published: 10 August 2009; accepted: 29 August 2009; published online: 30 September 2009.

Citation: Pelphrey KA, Lopez J and Morris JP (2009) Developmental continuity and change in responses to social and nonsocial categories in human extrastriate visual cortex. Front. Hum. Neurosci. 3:25. doi: 10.3389/neuro.09.025.2009

Copyright (C) 2009 Pelphrey, Lopez and Morris. This is an open-access article subject to an exclusive license agreement between the authors and the Frontiers Research Foundation, which permits unrestricted use, distribution, and reproduction in any medium, provided the original authors and source are credited. 